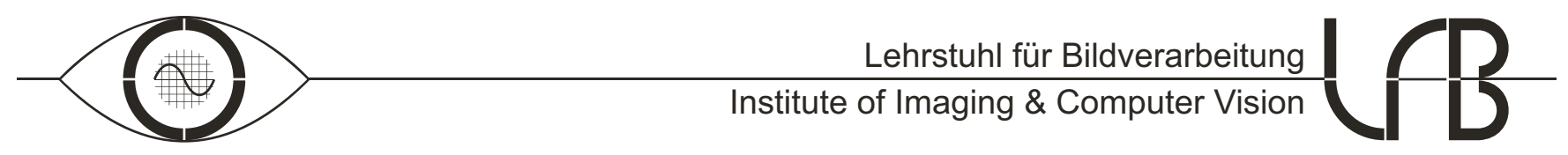

\title{
Design of Multi-Steerable Filters and their Application for the Detection of Corners and Junctions
}

\author{
Matthias Mühlich and Thorsten Dahmen and Til Aach \\ Institute of Imaging and Computer Vision \\ RWTH Aachen University, 52056 Aachen, Germany \\ tel: +49 2418027860 , fax: +49 2418022200 \\ web: www.lfb.rwth-aachen.de
}

in: IEEE International Conference on Image Processing (ICIP2007). See also BIBTEX entry below.

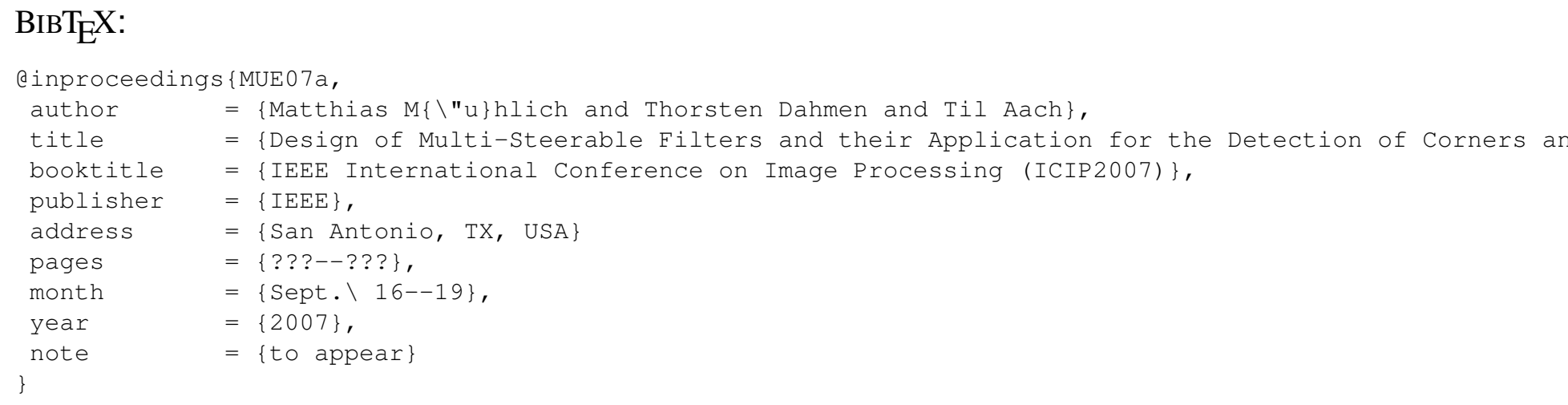

(C) 2007 IEEE. Personal use of this material is permitted. However, permission to reprint/republish this material for advertising or promotional purposes or for creating new collective works for resale or redistribution to servers or lists, or to reuse any copyrighted component of this work in other works must be obtained from the IEEE. 


\title{
DESIGN OF MULTI-STEERABLE FILTERS AND THEIR APPLICATION FOR THE DETECTION OF CORNERS AND JUNCTIONS
}

\author{
Matthias Mühlich, Thorsten Dahmen, and Til Aach \\ Institute of Imaging and Computer Vision, RWTH Aachen University, 52056 Aachen, Germany \\ $\{$ muehlich, dahmen, aach\}alfb.rwth-aachen. de
}

\begin{abstract}
The theory of steerable filters (introduced by Freeman and Adelson in 1991) offers a convenient and computationally efficient method to represent the rotation of a specific class of $2 \mathrm{D}$ filters as a linear combination of base filters. In this paper, we generalize this theory to filters which are modeled as point-by-point products of multiple (in our examples: two) base filters which each are rotated by individual rotation angles, thus introducing the term "multi-steerability".

While standard steerable filters are useful for the detection of edges or lines (oriented in some unknown direction), our novel extended theory can be applied to the detection of features which are characterized by multiple orientations, for instance corners or line crossings, which greatly increases the applicability of the theory. We conclude our paper with an example for detecting the crossings in a checkerboard image using double-steerable filters.
\end{abstract}

Index Terms - steerable filters, feature detection, orientation estimation

\section{INTRODUCTION}

Filtering an image with a set of rotated filter kernels is an important low level vision problem, applicable to a variety of different applications such as feature tracking, edge detection, image enhancement or texture analysis. The steerable filters approach developed by Freeman and Adelson [1] allows a convenient and fast implementation for such rotated filters, namely as a weighted sum of a limited number of linear base filters.

\subsection{A general view on steerability}

The special elegance of the steerable filter approach comes with the fact that the whole dependency on the rotation angle is encapsulated in the weighting coefficients (also termed "interpolation functions"), thus giving a linear representation for the non-linear action of rotation.

The obvious price one has to pay is the fact that not all possible filter kernels are steerable. However, the class of filters which can be steered contains many filters which are relevant for image analysis. In their seminal paper introducing steerable filters [1], Freeman and Adelson have already shown that all filters $h$ which can be represented in polar representation as

$$
h(r, \phi)=\sum_{n=-N}^{N} a_{n}(r) e^{j n \phi}
$$

are steerable. Equation (1) is a Fourier series of order $N$ in the angular function with weight coefficients $a_{n}(r)$ that are allowed to depend on the radius $r$. In other words, a filter is steerable if the number of Fourier coefficients is limited. This is the underlying reason why it is possible to synthesize any arbitrary rotation from a linear combination of a limited number of base filters, and additionally, the minimal number of base filters can be shown to be the number of non-zero Fourier coefficients.

In practice, however, several different ways of designing steerable filters exist. This is due to the fact that some authors desire Cartesian (i.e. $x y$ ) separable filters only (even if this usually goes at the expense of obtaining more than the minimal number of base filters $[1,2])$. Other authors optimize for phase-invariant behavior, that is, a similar (ideally: equal) filter response energy for both line and edge structures [3]. An important example for this approach are the steerable wedge filters introduced by Simoncelli et al. [4, 5].

The basic idea of steerable filters, namely representing a potentially complicated non-linear operation such as rotation as a linear combination of basic operators (which can be applied in a preprocessing step), can also be found for shearing, multiscale approaches or combination of such ideas which lead to the generic term deformable filters introduced in [6, 7]. Some authors like Michaelis and Sommer [8] and later Teo and Hel-Or [9] have even considered embedding the approach behind steerable filters in a Lie group theory framework. All these ideas have in common that the set of allowed operators must be reduced to a class which can actually be modeled as a weighted linear combination of a limited number of specific and fixed operators.

In this paper, we will present a different approach for an extension of steerable filters which is directly related to the detection of meaningful image features like junctions. This 
novel approach is based on the point-by-point multiplication of steerable filters. As steerable filters (with respect to rotation) are band limited in the angular function and because multiplication in the spatial domain means convolution in the Fourier domain, it is obvious that such product functions are angularly band limited, i.e., steerable, as well. More specifically, we will show in section 3 that such filters are now individually steerable with respect to two or more angles.

But first, we start with a short introduction to rotated matched filtering which is based on [2]. Then, we will discuss a new way of designing (single-)steerable filters by directly modeling template functions for image features like edges or lines as polar-separable function approximations in section 2. In section 3, we introduce the novel concept of multisteerability and also give this theoretical concept an actual meaning for image processing, when we show how to model multiply oriented image structures like corners or crossing using such filters. We conclude the paper with an example for detecting crossings in checkerboard images using doublesteerable templates in section 4 .

\subsection{Rotated matched filtering using steerable filters}

Let us assume we want to detect an image feature (e.g. an edge or ridge) in an image $s(\vec{x})$ at an unknown position and orientation. This detection problem can be formulated as rotated matched filtering. This means that we compute (and maximize) the correlation between rotated versions of a $2 \mathrm{D}$ feature template $s_{0}$ and the signal $s$. Let us define $h(\vec{x})=$ $s_{0}(-\vec{x})$ (such that correlation can be expressed as convolution) and let us introduce the rotation matrix $\mathbf{R}$; in two dimensions, it depends on a single angle $\phi$ :

$$
\mathbf{R}_{\phi}=\left(\begin{array}{cc}
\cos (\phi) & \sin (\phi) \\
-\sin (\phi) & \cos (\phi)
\end{array}\right)
$$

In slight abuse of notation, we denote the image template function as $h$, regardless whether it is represented in Cartestian or polar coordinates. Then $h\left(\mathbf{R}_{\phi} \vec{x}\right)$ is a rotated version of the (mirrored) feature template and the convolution result

$$
Q(\phi, \vec{x})=s(\vec{x}) * h\left(\mathbf{R}_{\phi} \vec{x}\right)
$$

indicates the strength of the sought feature for a given rotation angle $\phi$ (resp. rotation matrix $\mathbf{R}_{\phi}$ ) at position $\vec{x}$. Let $\hat{\phi}(\vec{x})$ be the rotation angle which maximizes $Q$ at a given position $\vec{x}$ and let $\hat{Q}(\hat{\phi}, \vec{x})$ denote this maximum value. Then $\hat{\phi}$ and $\hat{Q}$ are maximum likelihood estimates for orientation and feature strength for the signal model

$$
s(\vec{x})=Q s_{0}\left(\mathbf{R}_{\phi}\left(\vec{x}-\vec{x}_{0}\right)+\vec{x}_{0}\right)+n(\vec{x})
$$

where $n(\vec{x})$ denotes white Gaussian noise.

In general, this estimation scheme would require the implementation of many filters. For 2D estimation, rotation can be parameterized with a single angle, so it would require as many filters as quantization levels of the angle. This is possible, but impractical. To overcome this problem, one can restrict the allowed template functions to the class of steerable filters. In a recent paper, Jacob and Unser [2] show that many classical feature detectors like Canny's edge detector [10] or the eigendecomposition of the Hessian matrix (e.g. [11]) can be interpreted in the context of steerable filters in this way.

\section{SINGLE-STEERABLE FILTERS AND ORIENTATION ESTIMATION}

The most common approach for designing steerable filters, especially in introductory material like [12], is considering derivatives of some isotropic function $g(r)$ with respect to $x$ and $y$ (or $\phi=0$ and $\phi=\frac{\pi}{2}$ in polar representation) as base filters. For the most popular choice, the Gaussian function

$$
g(r)=e^{-c r^{2}}=e^{-c\left(x^{2}+y^{2}\right)}=e^{-c x^{2}} e^{-c y^{2}},
$$

these derivatives up to any arbitrary order additionally remain separable in the Cartesian $x y$-domain. Designing the base filters as such derivatives of isotropic functions automatically guarantees band limited angular functions and hence steerability, but there are two drawbacks. Firstly, computing all derivatives w.r.t. the canonical directions $x$ and $y$ does not yield a minimal set of base filters [1]. But there is also a second point which will become important for this paper: we somewhat lose the connection to the Fourier coefficients and the angular signal they implicitly represent.

While Jacob and Unser [2] propose filter design criteria based on measures for 'localization' or 'absence of false oscillations', we will directly base our filter design on the angular function and its Fourier coefficients; with these coefficients being responsible for the steerability property, it seems logical to optimize them directly given some signal model and this signal model is chosen such that it represents oriented image features like edges or lines.

Local orientations are an important low level feature for analyzing and understanding image data. The basis for the concept of orientations is the observation that the spatial variation of the gradient directions is in general much slower than the spatial variation of the image itself [3]. Therefore, the study of signal gradients led to the concept of local orientations in signals, and the most important image features which can be described using this model are edges and lines. For lines, we can furthermore distinguish between unimodal lines and bimodal lines, see fig. 1 for ideal templates of the three models.

We already stress here that gray level scaling will be meaningful later on. While steerable filtering itself is a linear operation, the generalization towards multi-steerability requires a non-linear step. Linear transformations have no effect on feature detection using steerable filters, but if we construct multi-steerable filters using some non-linear operation, then 

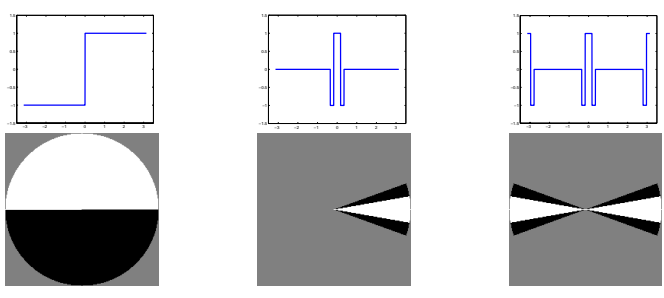

Fig. 1. From left to right, these images show the ideal templates for edge, unimodal line, and bimodal line. In the top row, the angular function is plotted between $-\pi$ and $\pi$ and the bottom row shows the resulting templates if all value larger than a specified radius $r_{0}$ are set to zero.

pre- and postprocessing with a linear gray level transformation (i.e., simple shifting and scaling) becomes an important tool for achieving certain desired properties. We therefore introduce the following convention: the range for the gray values shall be scaled from -1 (black) to 1 (white) with 0 (gray) denoting 'neutral' values. These three patterns can be expressed as polar separable functions using

$$
\begin{aligned}
h_{\text {edge }}(r, \phi) & =f(r) g_{\text {edge }}(\phi) \\
h_{\text {line1 }}(r, \phi ; \theta) & =f(r) g_{\text {line1 }}(\phi ; \theta) \\
h_{\text {line2 }}(r, \phi ; \theta) & =f(r) g_{\text {line } 2}(\phi ; \theta)
\end{aligned}
$$

with radial function

$$
f(r)=\left\{\begin{array}{ll}
1 & r<r_{\max } \\
0 & \text { else }
\end{array} .\right.
$$

Modeling non-constant functions (for instance exponential decay) is no problem as only the angular part needs to fulfill the steering condition; the radial part is just multiplicative. For the three signal models, we derive

$$
g_{\text {edge }}(\phi)=\left\{\begin{array}{cl}
1 & 0 \leq \phi<\pi \\
-1 & -\pi \leq \phi<0
\end{array}\right.
$$

for edges and

$$
\begin{aligned}
& g_{\text {line1 }}(\phi ; \theta)=\left\{\begin{array}{cl}
1 & |\phi| \leq \frac{\theta}{2} \\
-1 & \frac{\theta}{2}<|\phi| \leq \theta \\
0 & \text { else }
\end{array}\right. \\
& g_{\text {line } 2}(\phi ; \theta)=g_{\text {line } 1}(2 \phi ; 2 \theta)
\end{aligned}
$$

for the two line models. The orientation sensitivity of the line templates can be adjusted using the angle $\theta$. Fig. 2 shows Fourier series approximations to these base templates.

\section{DEFINING MULTI-STEERABLE FILTERS}

While being important low-level image features, singleoriented structures allow only localization along the orientation direction (aperture problem). Features better suited
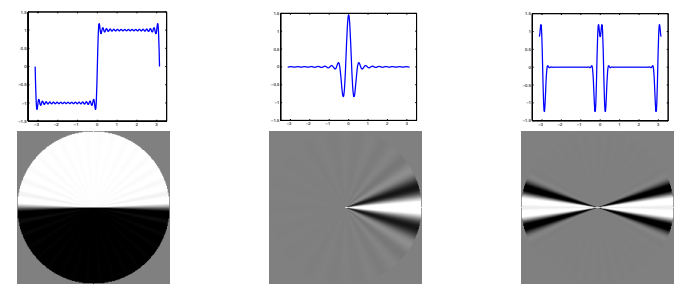

Fig. 2. Approximation of fig. 1 using a Fourier series. The order used here was 15 and the opening angle $\theta$ for the wedges representing lines was chosen to be $20^{\circ}$.
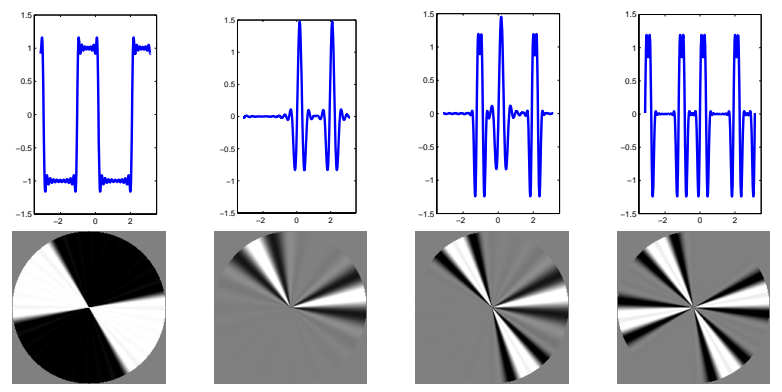

Fig. 3. Checkerboard pattern (edge $\times$ edge), L-junction (line1 $\times$ line1), T-junction (line $1 \times$ line2), $\mathrm{X}$-junction (line $2 \times$ line2). Angular function in top row, resulting feature template in bottom row. The three junctions also require a simple shift operation as pre- and postprocessing, see main text.

for purposes like tracking are those features showing multiple orientations, either occludingly or additively superposed. The theory of multiple orientation estimation can be found in $[13,14]$. However, the big advantage of steerable filters is that they are implicitly connected to an estimated signal model via their Fourier coefficients. Moreover, we can determine which type of double-oriented structure we actually see.

Considering the design of multi-steerable filters as products in the spatial domain, the scaling range of our singlesteerable filters from -1 to 1 immediately becomes clear: multiplication with 1 changes nothing, while multiplication with 0 cancels other signals out.

Multiplication of two rotated edge functions directly yields a checkerboard pattern, see fig. 3 . In the same figure, we also present the generation of L-, T- and $\mathrm{X}$-junctions using products between different line templates. The junction images, however, needed some additional clever linear pre- and postprocessing: add 1 (set gray area from 0 to 1, i.e., the neutral element w.r.t. multiplication, and the black area next to the line from -1 to 0 , i.e., to 'cancel out'), then multiply, then subtract 1 to set the DC level to zero again.

Obtaining the interpolation functions needed to steer the templates is elegant as well. Let $\vec{b}_{1}$ and $\vec{b}_{2}$ be vectors containing the coefficients for the single-steerable templates. Then 


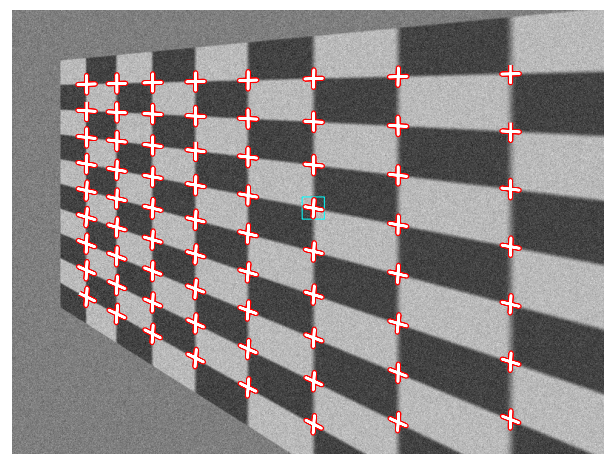

Fig. 4. Synthetic checkerboard image (with added Gaussian noise, SNR $=10 \mathrm{~dB}$ ) and detected checkerboard crossings. The rectangle shows the size of the double-steerable filter templates; here, $29 \times 29$ windows were used.

all the products between the individual elements form the set of double-steerable interpolation coefficients. Generalization towards more than two orientations, e.g. towards three unimodal lines for modeling Y-junctions is straightforward.

\section{REPRESENTING CHECKERBOARD CROSSINGS WITH DOUBLE-STEERABLE FILTERS}

In order to show the wide applicability of multi-steerable filtering for image analysis, we generated a synthetic image of a checkerboard and added Gaussian noise $(\mathrm{SNR}=10 \mathrm{~dB})$. The resulting image then is similar to those which are commonly used for camera calibration. Fig. 4 shows the image and the checkerboard crossings which were detected using a doublesteerable filter approach. For finding all 69 visible crossings in this $800 \times 600$ pixel image, our algorithm written in pure Matlab needed 17.5 seconds on a $3 \mathrm{GHz}$ dual Pentium computer. The application to real data (including highly distorted checkerboard images taken with an endoscope) is investigated further in [15].

\section{SUMMARY AND CONCLUSION}

In this paper, we presented the theory of multi-steerable filters and gave examples for the case of double-steerability. Just like standard steerable filters, this theory allows to represent templates as sums of base templates, but in contrast to (single) steerable filters, the templates computed with our novel theory can be steered in two (or potentially more) directions individually.

This greatly increases the class of templates which can be modeled with the new theory. Especially for image analysis, important features like corners, checkerboard patterns or various forms of line crossings now become available for feature detection based on steerable filtering.
Additionally, rotated matched filtering using such filters directly gives rise to line (both unimodal or bimodal) and edge orientations in the image. This stands in contrast to other multiple orientation estimation schemes, which are not connected to some assumed signal model and therefore do not allow a distinction between different junction types. Concluding this paper, we state that the whole theory is rather straightforward, yet very powerful and promising.

Matlab demonstration code for double-steerable filters can be downloaded at our homepage: www.lfb.rwth-aachen.de/en/highlights/ multi_steerable_filters.html.

\section{REFERENCES}

[1] W.T. Freeman and E.H. Adelson, "The design and use of steerable filters," IEEE Trans. PAMI, 13 [9], pp. 891-906, 1991.

[2] M. Jacob and M. Unser, "Design of steerable filters for feature detection using Canny-like criteria," IEEE Trans. PAMI, vol. 26, no. 8, pp. 1007-1019, 2004.

[3] G. Granlund and H. Knutsson, Signal Processing for Computer Vision, Kluwer, 1995.

[4] E.P. Simoncelli, "Design of multi-dimensional derivative filters," in Proc. ICIP 1994, vol. 1, pp. 790-794.

[5] E.P. Simoncelli and H. Farid, "Steerable wedge filters for local orientation analysis," IEEE Trans. Image Processing, vol. 5, no. 9, pp. 1377-1382, 1996.

[6] P. Perona, "Deformable kernels for early vision," IEEE Trans. PAMI, vol. 17, no. 5, pp. 488-499, 1991.

[7] R. Manduchi, P. Perona, and D. Shy, "Efficient deformable filter banks," IEEE Trans. Signal Processing, vol. 46, no. 4, pp. 1168-1173, 1998.

[8] M. Michaelis and G. Sommer, "A Lie group approach to steerable filters," Patt. Rec. Lett., 16: 1165-1174, 1995.

[9] P.C. Teo and Y. Hel-Or, "Design of multiparameter steerable functions using cascade basis reduction," Pattern Recognition Letters, vol. 21, no. 6, pp. 552-556, 1999.

[10] J. Canny, "A computational approach to edge detection," IEEE Trans. PAMI, vol. 8, no. 6, pp. 679-698, 1986.

[11] D. Eberly, R. Gardner, B. Morse, S. Pizer, and C. Scharlach, "Ridges for image analysis," Tech. Rep. TR93-055, Univ. of North Carolina, Chapel Hill, NC, USA, 1993.

[12] D. J. Heeger, "Notes on steerable filters," 1998.

[13] T. Aach, C. Mota, I. Stuke, M. Mühlich, and E. Barth, "Analysis of superimposed oriented patterns," IEEE Trans. Image Processing, vol. 15, no. 12, pp. 3690-3700, 2006.

[14] M. Mühlich and T. Aach, "A theory for multiple orientation estimation," in Proceedings European Conference on Computer Vision 2006, Horst Bischof and Aleš Leonardis, Eds. 2006, number 3952 in LNCS, pp. (II) 69-82, Springer.

[15] M. Mühlich and T. Aach, "High Accuracy Feature Detection for Camera Calibration: A Multi-Steerable Approach," in Proc. DAGM07: 29th Annual Symposium of the German Association for Pattern Recognition, Heidelberg, Germany, September 12-14, 2007, Springer, to appear. 